\title{
LASER-ATOM INTERACTIONS : RECENT THEORETICAL DEVELOPMENTS
}

\section{Jean DALIBARD and Claude COHEN-TANNOUDJI}

Laboratoire de Spectroscopie Hertzienne de 1'Ecole Normale Supérieure et Collège de France, 24 rue Lhomond, F 75231 Paris Cedex 05 France

In intense laser beams, when perturbative treatments are no longer valid, the dressed atom approach provides a quantitative understanding of the main features of dipole or intensity gradient forces (mean value, fluctuations, velocity dependence). In this lecture, we present such an approach and we apply it to atomic motion in an intense standing wave. New efficient laser cooling schemes taking advantage of stimulated processes are proposed. They work for a blue detuning and do not saturate at high intensity.

\section{INTRODUCTION}

During the last few years, several experiments have demonstrated that laseratom interactions provide the possibility to control the velocity ${ }^{1,2}$ and the position ${ }^{3}$ of an atom. A new exciting field of research is emerging which is called laser cooling and trapping ${ }^{4}$.

In order to introduce the subject of this talk, we consider first the simplest possible example of an atom irradiated by a laser plane wave with wave vector $\vec{k}$. When the atom absorbs a laser photon, the momentum gain is $\hbar \vec{k}$. If the atom falls back to the ground state by stimulated emission, the atomic momentum returns to its initial value. But, if the emission process is a spontaneous one, the momentum loss during such a process is zero by symmetry, because spontaneous emission can occur with equal probabilities in opposite directions. It follows that the net atomic momentum gain in a fluorescence cycle (absorption + spontaneous emission) is $h \vec{k}$. Consequently, the mean force $\langle\vec{F}\rangle$ experienced by the atom is given by

$$
\langle\vec{F}\rangle=\hbar \vec{k} \Gamma \sigma_{\text {ee }}
$$

where $\Gamma \sigma_{e e}$ is the mean number of fluorescence cycles per unit time, equal to the product of the population $\sigma_{\text {ee }}$ of the excited atomic state e by the spontaneous emission rate $\Gamma$, which is also the natural width of e. Such a force is nothing but the well known radiation pressure ${ }^{5-7}$ which has been used for slowing down atomic beams ${ }^{1}$. At high intensities, $\sigma_{\text {ee }}$ tends to $1 / 2$ and $\langle\vec{F}\rangle$ saturates to the value $\hbar \vec{k} \Gamma / 2$.

Suppose now that the atom is irradiated by two counterpropagating laser 
beams, with the same intensity, and with a frequency $\omega_{L}$ detuned to the red with respect to the atomic one $\omega_{0}$ (the detuning $\delta=\omega_{L}-\omega_{0}$ is negative). If the intensity of such a standing wave is weak enough, the radiation pressures of the two counterpropagating waves can be added independently. Because of the Doppler effect, the atom gets closer to resonance with the opposing wave, farther from resonance with the copropagating one. It is slowed down. This is the principle of usual radiative cooling 8,9 . Such a scheme leads to velocity damping times of the order of

$$
\tau_{0}=\hbar / R
$$

where

$$
R=\hbar^{2} k^{2} / 2 M
$$

is the recoil energy and $M$ the atomic mass, and to minimum kinetic temperatures $T$ of the order of

$$
\mathrm{k}_{\mathrm{B}} \mathrm{T}=\mathrm{\hbar} \Gamma / 2
$$

where $k_{B}$ is Boltzmann's constant ${ }^{10}$. For sodium atoms, $\tau_{0}$ is of the order of a few microseconds and $T$ is as low as $240 \mu \mathrm{K}$. The possibility of cooling atoms with such "optical molasses" has been recently demonstrated ${ }^{2,4}$.

If the intensity of the laser standing wave is increased (saturation parameter $s \gg 1$ ), the physical picture given above breaks down. Stimulated emission processes, responsible for a coherent redistribution of photons between the two counterpropagating waves, become predominant : the absorption of a photon in a plane wave can be followed by the stimulated emission of a photon in the counterpropagating wave, giving rise to a change of atomic momentum equal to $\pm 2 \hbar \vec{k}^{11,12}$. Such stimulated processes, which occur at a rate of the order of the Rabi frequency $\omega_{1}$, are at the origin of dipole forces or intensity gradient forces $^{5,6}$ (which have been recently used for trapping atoms ${ }^{3,4}$ ). In a strong standing wave, it has been shown theoretically that they produce a heating of the atoms for a red detuning $(\delta<0)$ and a cooling for a blue one $(\delta>0)^{5,13,14}$, contrarily to what happens in the "radiation pressure molasses" described above.

The purpose of this lecture is to present recent theoretical developments which have provided a simple physical interpretation of atomic motion in an intense laser wave, and in particular in an intense standing wave ${ }^{15}$. Such an interpretation is based on the dressed atom approach which is briefly recalled in section 2. We then show in section 3 how dipole or intensity gradient forces can be interpreted in terms of two valued dressed state dependent forces (optical Stern and Gerlach effect). The particular case of an intense standing wave is considered in section 4, and a new efficient laser cooling mechanism, which 
does not saturate at high intensity, is introduced ("Stimulated molasses"). Finally, possible applications of stimulated molasses are investigated in section 5 .

2. THE DRESSED ATOM APPROACH. CONNECTION WITH RESONANCE FLUORESCENCE ${ }^{16-18}$

In the high intensity limit, the Rabi frequency $\omega_{1}$, characterizing the strength of the laser-atom coupling, is large compared to the spontaneous emission rate $\Gamma$. We therefore consider in a first step the energy levels of the combined system atom + laser photons interacting together (dressed states). Then, in a second step, we take into account the coupling with the vacuum field which gives rise to spontaneous transitions between dressed states. Resonance fluorescence thus appears as spontaneous emission of radiation from the dressed atom.

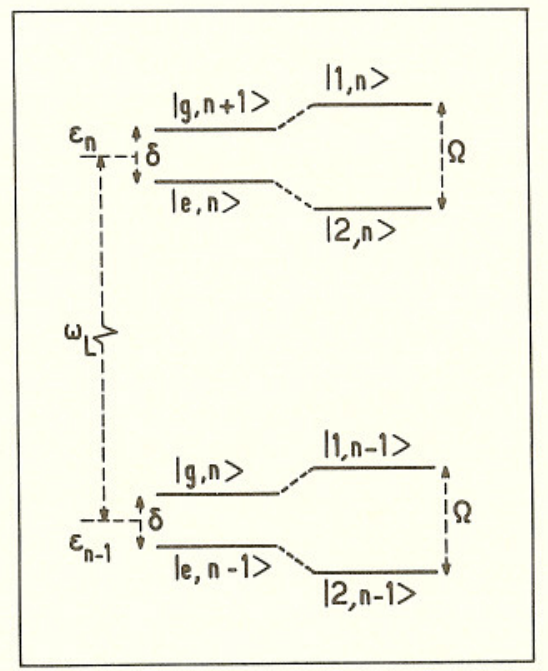

FIGURE 1

Left part : unperturbed states of the combined atom-laser photons system, in absence of coupling, bunched in well-separated two dimensional manifolds Right part : dressed states resulting from the atom-laser coupling

Let us first introduce the dressed states for a 2-1evel atom at rest in a given point $\vec{r}$. In absence of coupling, the energy levels of the combined system atom + laser photons are labelled by two quantum numbers, e or $\mathrm{g}$ for the atom (excited or ground state), $n$ for the number of laser photons. Such "unperturbed" states are represented on the left part on Fig. 1 . When the laser frequency $\omega_{L}$ is close to the atomic one $\omega_{0}$, these states are bunched into two dimensional manifolds... $\varepsilon_{n}=\{|g, n+1>, \quad| e, n>\}, \varepsilon_{n-1}=\{\mid g, n>, e, n-1>\} \ldots$, 
the distance between the two levels of a given manifold being $\hbar \delta=\left(\omega_{L}-\omega_{0}\right)$ and the distance between two adjacent manifolds being $\hbar \omega_{L}$. The laser-atom coupling $V$ connects the two states of a given manifold. For example, the atom in the ground state $\mathrm{g}$ and in presence of $\mathrm{n}+1$ laser photons can absorb one laser photon and jump into the excited state e. This means that $V$ has a non zero matrix element between the two states $\mid g, n+1>$ and $\mid e, n>$ of $\varepsilon_{n}$. Actually, one can show that

$$
<e, n|V| g, n+1>=\frac{\hbar}{2} \omega_{1}(\vec{r}) e^{i \varphi(\vec{r})}
$$

where $\varphi(\vec{r})$ is the phase of the laser field and $\omega_{1}(\vec{r})$ the Rabi frequency related to the amplitude $\vec{\varepsilon}_{\circ}(\vec{r})$ of the laser field and to the transition dipole moment $\overrightarrow{\mathrm{d}}$ by

$$
\hbar \omega_{1}(\vec{r})=-\vec{d} \cdot \vec{\varepsilon}_{0}(\vec{r})
$$

This coupling gives rise to two perturbed states, $\mid 1, n>$ and $\mid 2, n>$ (for $\varepsilon_{n}$ ), represented on the right part of Fig. 1. These dressed states are both 1 inear combinations of the unperturbed states $\mid e, n>$ and $\mid g, n+1>$ and are separated by a splitting $\hbar \Omega$ given by

$$
\Omega(\vec{r})=\left[\delta^{2}+\omega_{1}^{2}(\vec{r})\right] 1 / 2
$$

Consider now the effect of spontaneous emission. The emission frequencies correspond to transitions allowed between dressed levels, i.e. to transitions between states connected by a non zero matrix element of the atomic dipole operator D. In the uncoupled basis, D, which does not change the number of laser photons, connects only $\mid e, n>$ and $\mid g, n>$. Since both dressed states $\mid 1, n>$ and $\mid 2, n>$ of $\varepsilon_{n}$ are contaminated by $\mid e, n>$, and both dressed states $|1, n-1\rangle$ and $\mid 2, n-1>$ of $\varepsilon_{n-1}$ are contaminated by $\mid g, n>$, we find 4 allowed transitions between $\varepsilon_{n}$ and $\varepsilon_{n-1}$ : transition $|1, n>\rightarrow| 2, n-1>$ corresponding to a frequency $\omega_{L}+\Omega$, transition $|2, n>\rightarrow| 1, n-1>$ corresponding to a frequency $\omega_{L}-\Omega$, and transitions $|i, n>\rightarrow| i, n-1>$ (with $i=1,2$ ) corresponding both to a frequency $\omega_{L}$. The dressed atom approach thus provides a straightforward interpretation of the "fluorescence triplet" 19 emitted by a 2 level atom irradiated by a resonant laser beam. Similarly, various features of photon correlations observed on the fluorescence light can be easily understood by considering the sequence of fluorescence photons as being emitted in a "radiative cascade" by the dressed atom ${ }^{18}$.

3. DRESSED ATOM INTERPRETATION OF DIPOLE FORCES ${ }^{15}$

We apply now the dressed atom approach to the interpretation of dipole forces which are associated with the intensity gradients of the laser beam. 
In an inhomogeneous laser beam, the laser intensity is position dependent. It follows that $\omega_{1}^{2}(\vec{r})$, and consequently $\Omega(\vec{r})$ according to $(4)$, vary in space. In Fig. 2, we have represented the variations of the energies of the dressed states across a gaussian laser beam. Out of the laser beam, the dressed levels coincide with the bare ones, and their splitting is just $\hbar \delta$. Inside the laser beam, each dressed level $|1, n\rangle$ or $|2, n\rangle$ is a linear superposition of $|g, n+1\rangle$ and $\mid e, n>$ and the splitting between the two dressed states of a given manifold becomes $\hbar \Omega(\vec{r})$, which is larger than $\hbar \delta$.

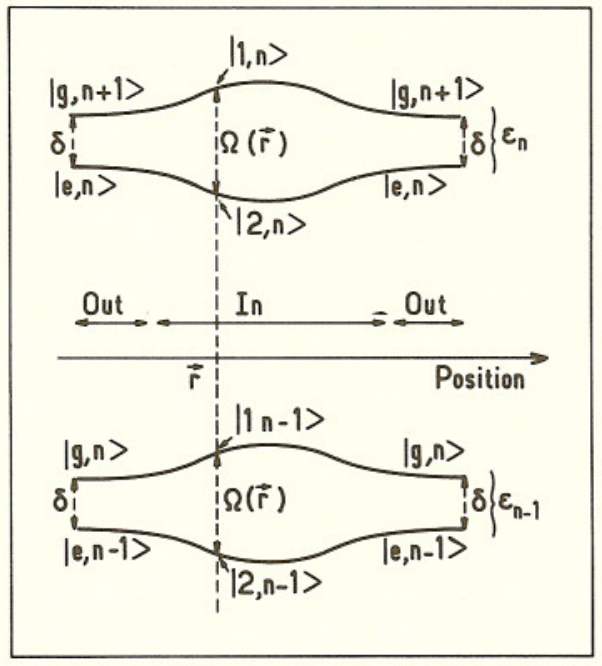

FIGURE 2

Variations across a gaussian laser beam of the dressed-atom energy levels. Out of the laser beam, the energy levels connect with the uncoupled states of Fig. 2, separated by $\delta$. In the laser beam, the splitting between the dressed states is $\Omega(\vec{r})>\delta$

Within each manifold, the energy diagram of Fig. 2 is similar to the one of a spin $1 / 2$ magnetic moment in an inhomogeneous static magnetic field. It follows that, in absence of spontaneous emission, we can define a two-valued dressed state dependent force, equal to minus the gradient of the dressed state energy

$$
\begin{aligned}
& \vec{f}_{1}=-\vec{\nabla} \mathrm{E}_{1 \mathrm{n}}(\vec{r})=-(\hbar / 2) \vec{\nabla} \Omega(\vec{r}) \\
& \vec{f}_{2}=-\vec{\nabla} \mathrm{E}_{2 n}(\vec{r})=+(\hbar / 2) \vec{\nabla} \Omega(\vec{r})=-\vec{f}_{1}
\end{aligned}
$$

As in the ordinary Stern and Gerlach effect, we have a force that depends on the internal state of the dressed atom, but the basic interaction occurs now 
between an optical dipole moment and an inhomogeneous laser electric field (optical Stern and Gerlach effect).

The effect of spontaneous emission is to produce, at random times, transitions between dressed states of type 1 and dressed states of type 2, or vice versa. This changes in a random way the sign of the instantaneous two valued dressed state dependent force. Such a picture of an instantaneous force switching back and forth between two opposite values provides a simple understanding of the mean value and of the fluctuations of dipole forces.

Consider first the mean force. It can be written as

$$
\left\langle\vec{F}_{\text {dip }}>=\vec{f}_{1} \Pi_{1}+\vec{f}_{2} \Pi_{2}=\vec{f}_{1}\left(\Pi_{1}-\Pi_{2}\right)\right.
$$

where $\pi_{i}$ is the proportion of time spent in a dressed state of type $i(i=1,2)$. For $\delta>0$ (case of Fig. 2), the unperturbed state $\mid g, n+1>$ is above $\mid e, n>$, since $\omega_{L}>\omega_{0}$, and the dressed state $|1, n\rangle$ connects to $|g, n+1\rangle$ out of the laser beam. It follows that $|1, n\rangle$ is less contaminated by $|e, n\rangle$ than $|2, n\rangle$, and is therefore more stable with respect to spontaneous emission, so that $\Pi_{1}>\Pi_{2}$, and the atom spends more time in dressed states of type 1 than in dressed states of type 2. We conclude that, for $\delta>0$, the sign of the mean force is the one corresponding to level $|1, n\rangle$ : the atom is expelled out of the high intensity regions. For $\delta<0$, the conclusions are reversed. The dressed state $|2, n\rangle$ connects to $|g, n+1\rangle$ out of the laser beam, is more populated than $|1, n\rangle$ and imposes its sign to the mean dipole force which attracts the atom towards the high intensity regions. Finally, for $\delta=0$, the 2 dressed states contain the same admixture of $|e, n\rangle$, are equally populated $\left(\Pi_{1}=\Pi_{2}\right)$, so that the mean force vanishes. We understand in this way why the variations of the mean dipole force (for an atom initially at rest) versus the detuning $\delta=\omega_{L}-\omega_{0}$ are of a dispersive type. The argument given above is not only qualitative but also quantitative. If one calculates $\pi_{1}$ and $\pi_{2}$ from the master equation giving the spontaneous transition rates between the various dressed states, and if one puts their values in (6), one gets the exact value of the mean dipole force, (to lowest order in $\left.\Gamma / \omega_{1}\right)^{15}$.

Similarly, by studying the correlation function of the instantaneous force switching back and forth between $\vec{f}_{1}$ and $\vec{f}_{2}=-\vec{f}_{1}$, it is possible to get a quantitative interpretation of the diffusion coefficient associated with the fluctuations of dipole forces ${ }^{5,15}$.

\section{DRESSED STATES IN A STANDING WAVE. STIMULATED MOLASSES}

We come back now to the problem mentioned at the end of the introduction concerning the importance of stimulated processes in a strong standing wave. Atomic motion in a strong standing wave has been studied by several 
authors $20,13,5,21,15$. We have recently proposed a physical picture, based on the dressed atom approach, and which provides some physical insight in this problem ${ }^{15}$. We present in this section a summary of this theoretical work.

In a plane standing wave, along $0 z$, the Rabi frequency $\omega_{1}(z)$ is a periodic function of $z$

$$
\omega_{1}(z)=\omega_{1} \cos k z
$$

It follows that the 2 dressed states of a given manifold oscillate periodically in space since their splitting is according to (4) and (7).

$$
\Omega(z)=\left[\omega_{1}^{2} \cos ^{2} k z+\left(\omega_{L}-\omega_{0}\right)^{2}\right]^{1 / 2}
$$

Fig. 3 represents these dressed states for a positive detuning (dashed 1 ines). At a node of the standing wave $(z=\lambda / 4,3 \lambda / 4 \ldots), \omega_{1}(z)$ vanishes and the two dressed states $|1, n\rangle$ and $|2, n\rangle$ respectively coincide with the unperturbed states $|g, n+1\rangle$ and $|e, n\rangle$, separated by $\delta$ (dotted lines). Out of a node, $\omega_{1}(z)$ is different from zero, the dressed states are linear combinations of $\mid g, n+1>$ and $\mid e, n>$ and their splitting is maximum at the antinodes $(z=0, \lambda / 2, \lambda \ldots)$ where $\omega_{1}^{2}(z)$ reaches its maximum value. Consider now the effect of spontaneous emission. As we have seen in section 2 above, an atom in level $\mid 1, n>$ or $\mid 2, n>$ can emit a spontaneous photon and decay to levels $|1, n-1\rangle$ or $|2, n-1\rangle$. The key point is that, in a standing wave, the various rates for such spontaneous processes vary in space because of the $z$ dependence of the wave functions. For example, if $\delta$ is positive and if the atom is in the level $|1, n\rangle$, its decay rate is zero at a node where $|1, n\rangle=|g, n+1\rangle$ and maximum at an antinode where the contamination of $|1, n\rangle$ by $|e, n\rangle$ is maximum. On the contrary, for an atom in level $|2, n\rangle$, the decay rate is maximum at the nodes where $|2, n\rangle$ coincides with $\mid e, n>$.

The previous considerations will allow us now to understand why an atom is slowed down in an intense standing wave, when $\delta$ is positive, contrarily to what happens in usual radiation pressure molasses (weak standing waves). We can for example follow the "trajectory" of a moving atom starting at a node of the standing wave, in level $|1, n+1\rangle$ (full lines of Fig. 3). Starting from this valley, the atom $\mathrm{climbs}$ uphill until it approaches the top (antinode) where its decay rate is maximum. It may jump either in level $\mid 1, n>$ (which does not change anything from a mechanical point of view) or in level $\mid 2, n>$, in which case the atom is again in a valley. It has now to climb up again until it reaches a new top (node) where $|2, n\rangle$ is the most unstable, and so on ... It is clear that the atomic velocity is decreased in such a process, since the atom sees on the average more "uphi11" parts than "downhill" ones. Such a scheme can be actually considered as a microscopic realization of the 
"Sisyphus myth" : every time the atom has climbed a hill, it may be put back at the bottom of another one by spontaneous emission and it has to climb up again.

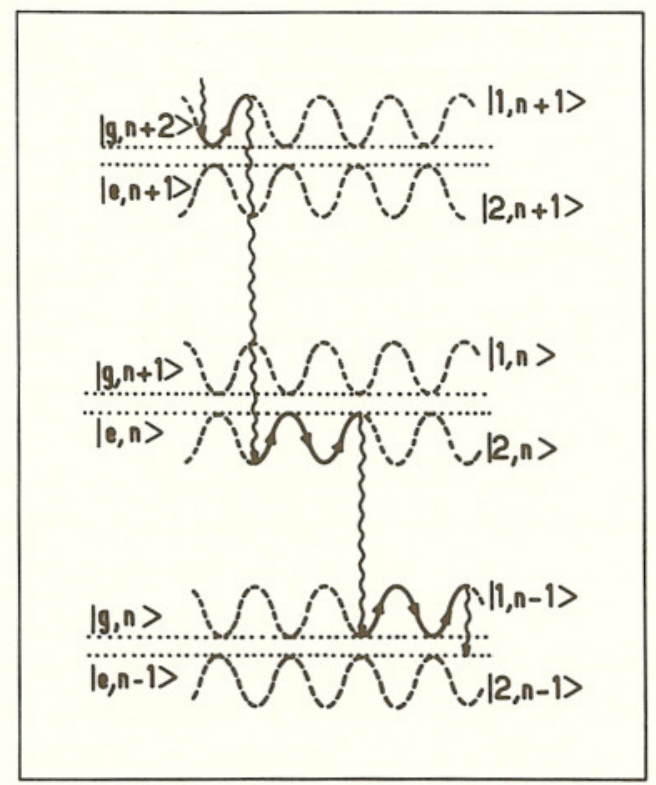

FIGURE 3

Laser cooling in a strong standing wave with a blue detuning $(\delta>0)$. The dashed lines represent the spatial variations of the dressed atom energy levels which coincide with the unperturbed levels (dotted lines) at the nodes. The full lines represent the "trajectory" of a slowly moving atom. Because of the spatial variation of the dressed wave functions, spontaneous emission occurs preferentially at an antinode (node) for a dressed state of type 1 (2). Between two spontaneous emissions (wavy lines), the atom sees on the average more uphill parts than downhill ones and is therefore slowed down.

We have used such a picture to derive quantitative results for the velocity dependence of the force acting upon the atom ${ }^{15}$. At very low velocities ( $k v \ll \Gamma$ ), we have a linear dependence with a slope which can be much higher than for usual radiation pressure molasses (by a factor of the order of $\omega_{1} / \Gamma$ ). The force reaches its maximum value for velocities such $k v \sim \Gamma$, or in other words, for situations in which, as in Fig. 3, the atom travels over a distance of the order of a wavelength between two spontaneous emissions. The important point is that the magnitude of this friction force is directly related to the modulation depth of the dressed energy levels, i.e. to the Rabi frequency $\omega_{1}$. As a consequence, this force increases indefinitely with the laser intensity. After this maximum, when $k v$ becomes large compared to $\Gamma$, the force decreases 
as $v^{-1}$ and finally, at very large velocities, resonances appear which are due to non-adiabatic Landau-Zener transitions between the two dressed states of each manifold 21 .

To conclude this section, it may be useful to analyze the energy-momentum balance in the cooling process associated with these "stimulated molasses". Between two spontaneous emission processes, the total (kinetic + potential) energy of the atom is conserved. When the atom climbs uphill, its kinetic energy is transformed into potential energy by stimulated emission processes which redistribute photons between the two counterpropagating waves at a rate $\omega_{1}$. Atomic momentum is therefore transferred to laser photons. The total atomic energy is then dissipated by spontaneous emission processes which carry away part of the atomic potential energy.

\section{APPLICATIONS}

\subsection{Transverse cooling of an atomic beam}

By irradiating at right angle an atomic beam with an intense standing wave detuned to the blue, it is possible to cool the transverse atomic velocities (along the direction of the standing wave) by the mechanism described in the previous section. If the transverse velocity spread $\Delta v$ is such that $k \Delta v \sim \Gamma$, the cooling efficiency is maximum and it is possible to cool the beam with an interaction length much shorter than the one required for usual radiation pressure molasses.

Actually, such an experiment has just been done in our group on a Cesium atomic beam ${ }^{22}$. An example of experimental results is represented in Fig. 4. Fig. 4a gives the transverse velocity profile of the atomic beam (measured by a hot wire detector located 2 meters downstream) in absence of laser. The velocity spread is $\pm 2 \mathrm{~m} / \mathrm{s}$. Fig. $4 \mathrm{~b}$ shows the effect of a laser standing wave detuned $+30 \mathrm{MHz}$ above resonance which corresponds to $\delta=+6 \Gamma$ (the power is $70 \mathrm{~mW}$, and the beam waist in the interaction region is $w=1,8 \mathrm{~mm}$ leading to $\omega_{1}=50 \Gamma$ ). The width of the velocity profile is 5 times narrower than the one of the unperturbed beam. Finally, if the laser frequency is detuned - $30 \mathrm{MHz}$ below resonance, the atomic beam is decollimated and exhibits a double peak structure (Fig. 4c).

Such an experiment demonstrates the efficiency of stimulated molasses. Realization of the same cooling with the usual radiation pressure molasses would have required an interaction length one order of magnitude larger. Furthermore, since stimulated forces do not saturate, the damping time for stimulated molasses, which is inversely proportional to $\omega_{1}$, could be yet more decreased, by increasing the laser power. 


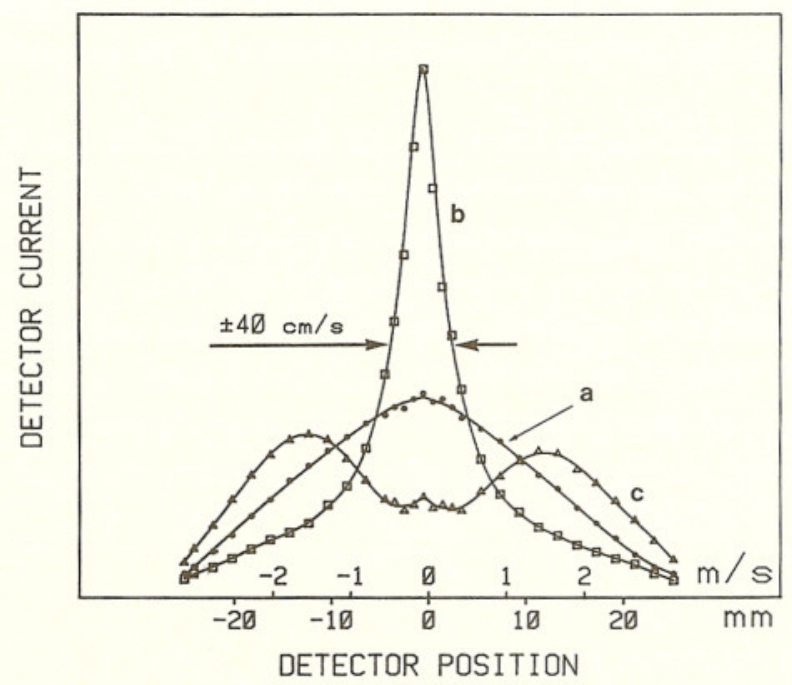

FIGURE 4

Transverse velocity profile of the Cs atomic beam

a - In absence of laser beam

b - In presence of an intense standing wave $\left(\omega_{1}=50 \Gamma\right)$ perpendicular to the atomic beam and detuned to the blue $(\delta=+16 \Gamma)$

c - Same conditions as b except for the detuning which is now negative $(\delta=-6 \Gamma)$

\subsection{Longitudinal slowing down of an atomic beam}

Suppose now that the atomic beam and the laser standing wave are paral1el. If the standing wave could be swept in order to have a weak enough relative velocity with respect to the atoms, corresponding to the maximum value of the decelerating force $(k v \sim \Gamma)$, it would be possible to slow down an atomic beam with an efficiency much higher than in experiments using radiation pressure. For Cesium, a laser intensity of $100 \mathrm{~mW} / \mathrm{mm}^{2}$ would allow to reduce the stopping distance from 1 meter to 10 centimeters. This might be of special interest for the realization of a compact atomic clock.

The realization of such an experiment would require two counterpropagating laser beams with frequencies $\omega+\Delta \omega$ and $\omega-\Delta \omega$ (in order to have a standing wave moving with a velocity $c \Delta \omega / \omega)$. The frequency offset $\Delta \omega$ should also vary in time (in order to have a standing wave moving with the decelerating atoms). The development of high power single mode laser diodes could provide a simple way to realize such an experimental scheme. 


\section{REFERENCES}

1) For a review of these experiments, see for example W.D. Phillips, J.V. Prodan and H.J. Metcalf, J.0.S.A. B 2 (1985) 1751 (Special issue on mechanical effects of light).

2) S. Chu, L. Hollberg, J.E. Bjorkholm, A. Cable and A. Ashkin, Phys. Rev. Let. 55 (1985) 48.

3) S. Chu, J.E. Bjorkholm, A. Ashkin and A. Cable, Phys. Rev. Let. 57 (1986) 314 .

4) S. Chu, Demonstration of laser cooling and trapping of atoms, this volume.

5) J.P. Gordon and A. Ashkin, Phys. Rev. A 21 (1980) 1606.

6) R.J. Cook, Comments At. Mol. Phys. 10 (1981) 267.

7) V.S. Letokhov and V.G. Minogin, Phys. Rep. 73 (1981) 1.

8) T.W. Hänsch and A. Schawlow, Opt. Commun. 13 (1975) 68.

9) D.J. Wineland and H.G. Dehmelt, Bul1. Am. Phys. Soc. 20 (1975) 637.

10) D.J. Wineland and W.M. Itano, Phys. Rev. A 20 (1979) 1521.

11) E. Kyrölä and S. Stenholm, Opt. Commun. 22 (1977) 123.

12) A.F. Bernhardt and B.W. Shore, Phys. Rev. A 23 (1981) 1290.

13) V.G. Minogin and 0.T. Serimaa, Opt. Commun. 30 (1979) 373.

14) V.G. Minogin, Opt. Commun. 37 (1981) 442.

15) J. Dal ibard and C. Cohen-Tannoudji, J.0.S.A. B 2 (1985) 1707.

16) C. Cohen-Tannoudji and S. Reynaud, Dressed atom approach to resonance fluorescence, in : Multiphoton Processes, eds. J.H. Eberly and P. Lambropoulos (Wiley, New York, 1978) 103-118.

17) C. Cohen-Tannoudji and S. Reynaud, J. Phys. B 10 (1977) 345.

18) S. Reynaud, Ann. Phys. (Paris) 8 (1983) 315.

19) B.R. Mollow, Phys. Rev. 188 (1969) 1969.

20) A.P. Kazantsev, Zh. Eksp. Teor. Fiz. 66 (1974) 1599 (Sov. Phys. JETP 39 (1974) 784).

21) A.P. Kazantsev, V.S. Smirnov, G.I. Surdutovich, D.0. Chudesnikov and V.P. Yakovlev, J.O.S.A. B 2 (1985) 1731.

22) A. Aspect, J. Dalibard, A. Heidmann, C. Salomon and C. Cohen-Tannoudji submitted for publication. 